\title{
Standing on the ledge: atrial fibrillation ablation in patients with atrial septal closure devices
}

\author{
Marco Schiavone ${ }^{1}$ and Giovanni Battista Forleo ${ }^{1}$ \\ ${ }^{1}$ Luigi Sacco University Hospital
}

June 9, 2020

Percutaneous atrial septal defect (ASD) closure is the mainstay treatment for ostium secundum ASD and patent foramen ovale ${ }^{1}$. Patients with ASD may develop atrial fibrillation (AF), mostly due to structural atrial remodeling creating the substrate for macroreentry ${ }^{2,3}$. Timing of ASD closure is crucial to prevent further development of electrophysiological heterogeneity, thereby reducing morbidity associated with AF, even though patients with ASD closure devices remain at high risk of developing $\mathrm{AF}^{4}$.

The rising number of patients undergoing percutaneous ASD closure poses a new challenge in the treatment of coexistent AF. Furthermore, the reduction of surgical ASD treatment with concurrent cryo- or radiofrequency ablation (modified Maze procedure) is contributing to increase the number of patients who would benefit from catheter ablation after transcatheter ASD closure. Although some studies have shown a high acute success rate of catheter ablation in this population ${ }^{5}$, this treatment is often denied due the higher perceived risk of performing the transseptal puncture (TSP) after percutaneous repair of the defect.

Given the lack of definitive data on this topic, in this issue of the Journal, Garg et al. performed the first meta-analysis evaluating the safety and the efficacy of catheter ablation for AF in this subset of high-risk patients with ASD closure devices.

\section{Crossing the line: different techniques, same results.}

The investigators examined three different studies (Santangeli et al. ${ }^{6}$, Li et al. ${ }^{7}$ and Sanget al. ${ }^{8}$ ) including a total of 64 patients. The follow-up duration ranged from 6 to 22 months, and three different types of devices were utilized (Amplatzer, Abbott Medical, Plymouth, Minnesota ; CardioSEAL@, NMT Medical, Inc., Boston, Massachusetts ; Lifetech septal occluders, Lifetech Scientific, Shenzhen, China ). Acute procedural success (elimination of all pulmonary vein potentials and non-pulmonary vein triggers) was reached in $98.4 \%$ of patients (in 1 patient mitral isthmus block was not effective), showing a global feasibility of catheter ablation procedures in this population. The transseptal access was obtained through the native septum in the majority of patients (81.3\%); direct puncture of the device was safe but resulted in a significant prolongation of procedural times. Two strategies were used to facilitate the access into the left atrium through a septal device: Santangeli et al. ${ }^{6}$ placed an extra-stiff guidewire (0.032 inch) in a left pulmonary vein and advanced an upsized dilator $(11 \mathrm{Fr})$ over the wire to further dilate the access site across the device, Li et al. ${ }^{7}$ and Sang et al. ${ }^{8}$ advanced an angioplasty guide wire in the left superior pulmonary vein, withdrew the $8 \mathrm{Fr}$ dilator, and performed sequential dilatations using a $2.5-5.0 \mathrm{~mm}$ non-compliant balloon under pressure of 12-18 atm to ease sheath manipulation. Current data do not show differences in the two approaches; sequential dilations may reduce the mechanical force that theoretically can result in device dislodgment, although no such event was reported by Santangeli et al. ${ }^{6}$.

Neo-endothelialization and fibrous incorporation of the ASD closure device are generally completed within 1 to 3 months and, as described in a swine model $^{9}, 100 \%$ of the ASDs are generally closed at 3 months. Median 
times from ASD closure to catheter ablation in the three cohorts were 46 (6-82), 16 (6-36), and 11 (6-72) months respectively and this, apart from TSP techniques and operator skills, may have had a crucial role in determining the absence of device dislodgement, implying that catheter ablation might be safe at least 3 to 6 months after percutaneous ASD closure. Robust data on safety of direct puncture through other common

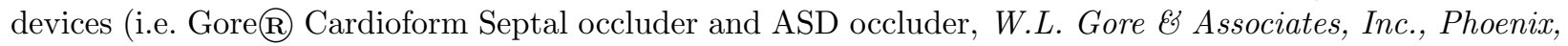
Arizona ) are lacking and should be investigated, although an early animal experience seems encouraging since left atrial access was achieved in sixteen canines without acute complications ${ }^{10}$.

\section{Is ICE the key to success?}

Both the aforementioned approaches seem safe and feasible, although it has to be underlined that 3 patients $(4.7 \%)$, all in the same cohort, ${ }^{7}$ had an inadvertent epicardial puncture during the transseptal access. In these 3 cases, although the device diameter ranged from 28 to $34 \mathrm{~mm}$, a non-echocardiography guided TSP across the native interatrial septum (posteroinferior to the ASD closure device) was performed. After the epicardial puncture, 2 patients underwent TSP across the device in the same procedure, while in one case the procedure was rescheduled under transesophageal echocardiography (TEE) guidance. This percentage of epicardial puncture during a fluoroscopy-guided transseptal approach is extremely high (3 out of 9 patients), especially if we consider that procedures were performed in a high-volume center by skilled operators. As stated by Santangeli et al.$^{6}$, intracardiac echocardiography (ICE) guidance is crucial to identify the portion of the native septum not covered by the ASD closure device (generally posteroinferior to the device), that should be preferentially targeted during TSP. In their cohort, if ICE failed to recognize a portion of the interatrial septum not covered by the device, mostly due to oversizing with respect to the interatrial septum or in cases with a device diameter [?] 26mm, a direct puncture of the closure device was performed. Although Sang et al. ${ }^{8}$ reported no procedural complications without ICE, suggesting that real-time monitoring of catheter movement may be sufficient to assess the optimal puncture site even when the device diameter is [?] $26 \mathrm{~mm}$, in our opinion ICE guidance remains crucial to improve safety and to avoid unnecessary attempts to TSP by identifying unfavorable anatomies in which a straight puncture of the device should be performed. Real-time 3D-TEE has also shown to provide an accurate anatomic definition of the native septum during $\mathrm{TSP}^{11}$ and may represent a valuable alternative, although ICE-guidance is corroborated by more robust data in this scenario and its use should be encouraged in routine practice.

\section{Procedural outcomes: the evidence is growing.}

Interestingly, in this issue of the Journal, Garg et al. showed that no significant differences in clinical and procedural outcomes were observed comparing septal vs. device puncture, apart from total procedural time that was significantly longer with TSP via the closure device vs. the native septum $(\mathrm{p}=0.004)$, mostly due to longer time needed to perform TSP. This suggests a similar maneuverability of the catheters in left atrium with both approaches. Recurrence of AF was not significantly different in the two groups and was comparable to AF ablation outcomes in patients without ASD closure device. As expected, long-term freedom from AF was higher in paroxysmal and persistent AF, while long-standing persistent AF patients were more unlikely to maintain sinus rhythm during follow-up. Considering the high success-rate in the early stages of AF, timing of catheter ablation is crucial and a "wait-and-see" strategy with a prolonged drug treatment ${ }^{12}$ is probably not worth it for ASD patients.

Of note, despite different follow-up strategies no residual interatrial shunt was reported in the three cohorts. In a medium-term follow-up (3 to 6 months after the procedure), Santangeli et al.$^{6}$ relied on contrast transthoracic echocardiography (TTE), with and without Valsalva maneuvers, Li et al. ${ }^{7}$ utilized TEE. Sang et al. ${ }^{8}$ relied only on non-contrast enhanced TTE, hence we cannot exclude that in this cohort the presence of a residual interatrial shunt might have been missed in some cases. Post-procedural shunts at ICE and contrast TTE were noted in a non-negligible number of patients in the first cohort ${ }^{6}$; therefore timing and the best strategy for evaluating residual shunts are of great importance. Follow-up in cases with direct puncture of the ASD closure device is even more challenging, because of the so-called "shadow effect" that might hamper a correct "anatomical" detection of the residual shunt. In our opinion, use of bubble-contrast TTE, enhanced with novel 3D imaging techniques, may be the best follow-up strategy in these patients, minimizing 
the risks of an incorrect detection of residual shunt, as well as avoiding discomfort of TEE (Figure 1) .

To sum up, despite limited data and a relatively small sample size, this meta-analysis highlights the feasibility and safety of catheter ablation in patients with ASD closure devices. In our opinion, these procedures should be centralized at high-volume centers; use of ICE should be encouraged, and a thorough follow-up with contrast-enhanced echocardiography should be performed. Garg et al. should be applauded for their work, useful to shed some light on this challenging population, establishing that AF ablation in patients with percutaneous atrial septal closure devices is safe and effective, but in the right hands.

\section{REFERENCES}

1. Baumgartner H, Bonhoeffer P, De Groot NMS, et al. ESC Guidelines for the management of grown-up congenital heart disease (new version 2010). Eur Heart J . 2010;31(23):2915-2957. doi:10.1093/eurheartj/ehq249

2. Blake GE, Lakkireddy D. Atrial Fibrillation and Atrial Septal Defect.J Atr Fibrillation . 2008;1(3). doi:10.4022/jafib.v1i1.390

3. Tse HF, Pelosi F, Oral H, Knight BP, Strickberger SA, Morady F. Effects of simultaneous atrioventricular pacing on atrial refractoriness and atrial fibrillation inducibility: Role of atrial mechanoelectrical feedback. J Cardiovasc Electrophysiol . 2001;12(1):43-50. doi:10.1046/j.1540-8167.2001.00043.x

4. Gatzoulis MA, Freeman MA, Siu SC, Webb GD, Harris L. Atrial arrhythmia after surgical closure of atrial septal defects in adults. $N$ Engl $J$ Med . 1999;340(11):839-846. doi:10.1056/NEJM199903183401103

5. Wang H, Wang C, Chen J, Zhao L, Pan X. Long-term outcome of catheter ablation for atrial tachyarrhythmias in patients with atrial septal defect. J Interv Card Electrophysiol . 2019;54(3):217-224. doi:10.1007/s10840-018-0505-1

6. Santangeli P, Di Biase L, Burkhardt JD, et al. Transseptal access and atrial fibrillation ablation guided by intracardiac echocardiography in patients with atrial septal closure devices. Heart Rhythm . 2011;8(11):16691675. doi:10.1016/j.hrthm.2011.06.023

7. Li X, Wissner E, Kamioka M, et al. Safety and feasibility of transseptal puncture for atrial fibrillation ablation in patients with atrial septal defect closure devices. Heart Rhythm . 2014;11(2):330-335. doi:10.1016/j.hrthm.2013.11.011

8. Sang CH, Dong JZ, Long DY, et al. Transseptal puncture and catheter ablation of atrial fibrillation in patients with atrial septal occluder: Initial experience of a single centre. Europace . 2018;20(9):1468-1474. doi:10.1093/europace/eux282

9. Sharafuddin MJA, Gu X, Titus JL, Urness M, Cervera-Ceballos JJ, Amplatz K. Transvenous closure of secundum atrial septal defects: Preliminary results with a new self-expanding nitinol prosthesis in a swine model. Circulation . 1997;95(8):2162-2168. doi:10.1161/01.CIR.95.8.2162

10. Anderson JH, Adamson T, Migliati E, et al. Perforating the GORE(r) CARDIOFORM septal occluder and atrial septal defect occluder to gain access to the left atrium. Catheter Cardiovasc Interv . 2020. doi:10.1002/ccd.28884

11. Mitacchione G, Curnis A, Forleo GB. Transseptal catheterization of the native septum for atrial fibrillation ablation in presence of septal occluder device: a novel approach with real-time 3D transesophageal echocardiographic guidance. J Interv Card Electrophysiol . 2020. doi:10.1007/s10840-020-00703-3

12. Mohanty S, Di Biase L, Mohanty P, et al. Effect of periprocedural amiodarone on procedure outcome in patients with longstanding persistent atrial fibrillation undergoing extended pulmonary vein antrum isolation: Results from a randomized study (SPECULATE). Heart Rhythm . 2015;12(3):477-483. doi:10.1016/j.hrthm.2014.11.016 
Patients with ASD closure devices undergoing AF catheter ablation

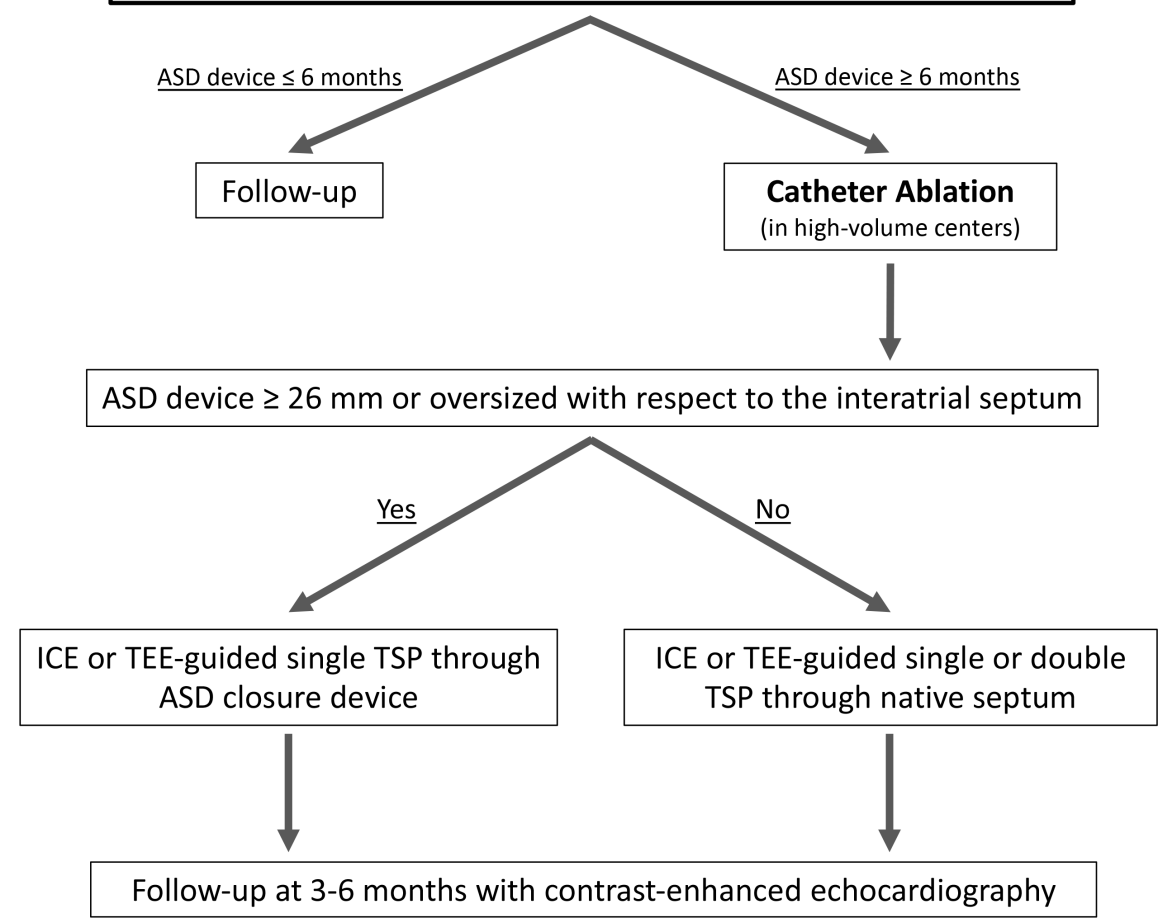

